\title{
Fast Neutron Imaging at a Reactor Beam Line
}

\author{
R. Zboray ${ }^{1, a^{*}}$, Ch. Greer ${ }^{1, b}$, A. Rattner ${ }^{1, c}$, R. Adams ${ }^{2, d}$, Z. Kis ${ }^{3, e}$ \\ ${ }^{1}$ Department of Mechanical and Nuclear Engineering, The Pennsylvania State University, 233 \\ Reber Building, University Park, PA 16802, USA \\ ${ }^{2}$ Swiss Federal Institute of Technology Zurich, Department of Mechanical and Process \\ Engineering, Sonnegstrasse 3, CH-8092, Zurich, Switzerland \\ ${ }^{3}$ Hungarian Academy of Science, Centre for Energy Research, 29-33 Konkoly Thege Miklos \\ street, 1121 Budapest, Hungary \\ arzz65@psu.edu, bczg5155@gmail.com, casr20@psu.edu, dadams@lke.mavt.ethz.ch, \\ dzkis@iki.kfki.hu
}

Keywords: Fast Neutron Imaging, Reactor Beam Line, Plastic Scintillator, ZnS

\begin{abstract}
Though fast neutron contribution in a thermal imaging beam line is typically considered as a burden, we have investigated its application for imaging using the high energy tail of the fission spectrum at a reactor beam line. Fast neutron imaging is a promising nondestructive technique for testing dense and voluminous objects of practically any material composition. Fast neutron radiography and tomography have been performed using the RAD beam line of the $10 \mathrm{MW}$ research reactor of the Budapest Neutron Centre (BNC), Hungary, using a camera-based imaging detector system on different bulky objects (up to $150 \mathrm{~mm}$ in diameter) and the results are presented here.
\end{abstract}

\section{Introduction}

Fast neutron imaging is a promising non-destructive technique for testing dense and voluminous objects of practically any material composition. Only fast neutrons can provide images with reasonable contrast and quality for dense and voluminous samples containing mixed low-Z/high$\mathrm{Z}$ materials, where photon-based techniques or even thermal neutron imaging would fail. Several different applications have been attempted ranging from homeland/civil security problems like detecting heavily shielded explosives, or contraband, to looking at robust cultural heritage objects or at industrial processes in heavily attenuating containers.

While fast neutron contribution in a thermal imaging beam line is typically considered as a burden, here we investigate its application for imaging. In many thermal neutron imaging beam lines, the epithermal and fast neutrons are intentionally suppressed by using, typically, sapphire filters to lower the dose to sensitive equipment such as the camera of the imaging detector, thereby also prolonging their functioning life time. In such beams, usually there is a significant fraction of $\mathrm{MeV}$ neutron content from the high-energy tail of fission or spallation spectrum of the source. Here, we focus on the application of reactor-based thermal beam lines for fast neutron imaging. This approach may be particularly useful as very few beam lines exist that are specially designed and dedicated to fast neutron imaging (e.g. at the YAYOI fast reactor in Japan [1] or the NECTAR beam line at the 20-MW FRM II reactor in Munich, Germany [2]). To our knowledge, besides our attempts in [3] and [4], no application of fast neutron imaging at a thermal beam line has been reported in the literature.

\section{Experimental setup}

The imaging beam line. We have primarily used the RAD beamline of the $10 \mathrm{MW}$ research reactor of the Budapest Neutron Centre (BNC), Hungary, to demonstrate the feasibility of fast 
neutron radiography and tomography [4]. The beamline is routinely utilized for thermal neutron imaging having a thermal flux of around $4 \times 10^{7} \mathrm{n} / \mathrm{cm}^{-2} \mathrm{~s}^{-1}$. The beam features a relatively high gamma background of $8.5 \mathrm{~Gy} / \mathrm{h}$ (non-attenuated) and a significant fast neutron contribution of $2.7 \times 10^{7} \mathrm{n} / \mathrm{cm}^{-2} \mathrm{~s}^{-1}(\mathrm{E}>2.5 \mathrm{MeV})$. These values have been carefully evaluated by the instrument crew. The fast flux specifically has been measured by the standard procedure, activating a Cdclad $\mathrm{Ni}$ foil utilizing the Ni-58(n,p)Co-58 threshold reaction $(\sim 2.5 \mathrm{MeV})$ as described in [9]. Further details about the beam line are given in [5]. We have used a $10 \mathrm{~mm}$ thick borated rubber mat filter (MirroBor), enabling the suppression of practically the entire thermal neutron component of the beam to avoid unnecessary sample activation. We also used a $200 \mathrm{~mm}$ thick lead sheet to filter direct in-beam gamma contribution, the efficacy of which as has been carefully determined using the shadow cone technique [3]. Most of the relatively modest gamma background in the experimental hatch is originating from prompt gammas due to activation of surrounding structures by scattered neutrons [3]. Details on how the beam filtering has been optimized to suppress the thermal component and maximize the fast neutron to gamma ratio are given in $[3,4]$. The filtering decreases the useful fast neutron flux to around $3.3 \times 10^{5} \mathrm{n} / \mathrm{cm}^{-2} \mathrm{~s}^{-1}$. The primary aperture of the beamline (situated at the boundary of the biological shield and the core reflector) with a diameter of $28 \mathrm{~mm}$ results in a calculated L/D of 177 at the position of the detector, which is placed at $4960 \mathrm{~mm}$ from the primary aperture, enabling a quasi-parallel beam imaging geometry.

The fast neutron imaging detector and scintillator. An ANDOR Neo $5.5(2560 \times 2160$ pix $)$ sCMOS camera with a $50 \mathrm{~mm}, \mathrm{f} / 1.2$ objective with a total field of view (FOV) of the detector of about $270 \times 230 \mathrm{~mm}^{2}$ at an effective pixel size of about $106 \mu \mathrm{m}$ was used. Regarding the scintillator screen, it should be pointed out that the most efficient nuclear reaction to convert fast neutrons to a detectable particle is elastic scatter on hydrogen (recoil protons). Therefore, hydrogen rich materials are preferred for fast neutron detection. Specifically, for imaging purposes, two main types of fast neutron scintillators are commonly used as practical choices: organic (plastic) slab scintillators and inorganic (mostly ZnS type) scintillator embedded in a transparent organic matrix used for conversion. We have tested both types of scintillators, a BC400 plastic scintillator slab from St. Gobain [6] and a commercially available scintillator with $\mathrm{ZnS}: \mathrm{Cu}$ mixed into a polypropylene (PP) matrix [7]. Both types of scintillators have their advantages and disadvantages. The plastic scintillator is transparent to its own light therefore larger thicknesses, up to e.g. a centimeter, can be applied even in slab form. This can improve the conversion efficiency which is important for highly penetrating, few $\mathrm{MeV}$ fast neutrons. However, a very large screen thickness has an adverse effect on the spatial resolution (e.g. proper focusing on a thicker screen becomes increasingly a problem). The most severe disadvantage of plastic scintillators compared to ZnS-based scintillators is the roughly factor ten lower light yield. This is especially detrimental for camera-based imaging detectors for which the light collection efficiency is relatively poor due mainly to the small solid angle under which the screen is seen by the objective, amounting to typically a factor of about $1 \mathrm{e}-4$. Therefore, if one uses a scintillator with relatively low light yield in such a setup, neutron collision events can go undetected. This we have pointed out in our earlier work using an 8mm thick BC400 [3]. Furthermore, thick plastic scintillator slabs have a higher gamma sensitivity, while thinner ZnSbased screens are somewhat less sensitive to gammas, on the order of half [3]. On the other hand, a serious drawback of ZnS-based screens is their low transparency due to the polycrystalline ZnS and the granular structure of the screen, which prohibits benefiting from higher thicknesses ( it is typically limited to about 2-4 mm), opposed to transparent screens. The diffuse scatter of the scintillation light in $\mathrm{ZnS}$-based screens is the main contributor to the native screen blur. Our 
findings clearly prove that by far the best imaging efficiency can be achieved using a ZnS-based screen for fast neutrons and that the compromise on the spatial resolution is not that severe, as is shown in [4].

\section{Results}

We have imaged different objects with increasing complexity as reported in [3,4], with a determined spatial resolution of approximately $1.6 \mathrm{~mm}$. The utility of fast neutron imaging has been demonstrated with the application to more complex and robust objects including a $150 \mathrm{~mm}$ diameter viscous coupling used in helicopter blade shafts [4]. Here we report on the latest investigations on a complex, robust object: a lithium-fueled power source for planetary landers Li-fueled reactor for conceptual planetary lander power system. Another complex object we imaged using fast neutrons was a proof-of-concept Li-fueled batch reactor for powering a planetary lander, that was developed and tested at Pennsylvania State University [8], Fig. 1a. This device would react stored lithium fuel with readily accessible in situ oxidizers on planetary surfaces to generate heat and power. This technology has been proposed for Venus surface missions, using the primarily $\mathrm{CO}_{2}$ atmosphere as an oxidizer, for Lunar and Mars missions (using in situ water and/or $\mathrm{CO}_{2}$ ), and for missions to Ocean Worlds such as Jupiter's moon Europa (using surface ice as an oxidizer). In a batch configuration, condensed Li-based products (e.g., $\mathrm{Li}_{2} \mathrm{C}_{2}, \mathrm{Li}_{2} \mathrm{CO}_{3}, \mathrm{Li}_{2} \mathrm{O}, \mathrm{LiOH}, \mathrm{LiH} . .$. ) would be retained in the reactor vessel, avoiding contamination of the surroundings. This technology may enable very high specific-energy power systems because only the low-mass $\mathrm{Li}$ fuel, and not the more massive oxidizer, must be transported with the lander [8].

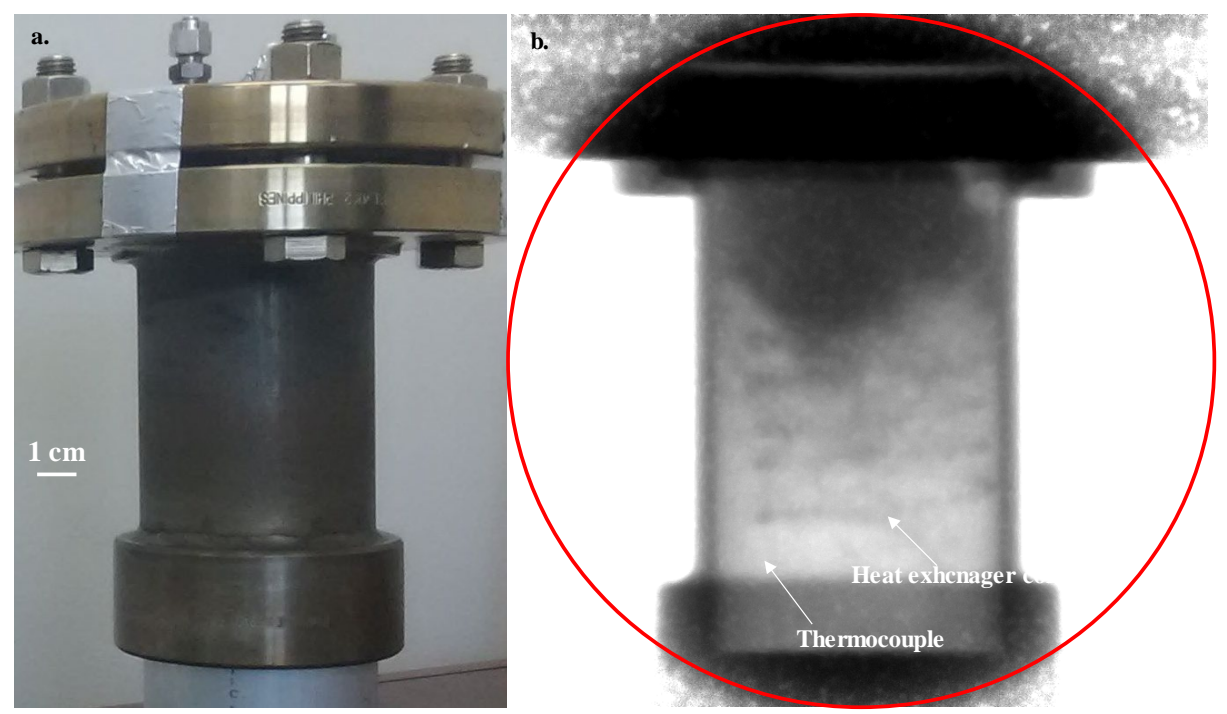

Figure 1: Photo (a) and a fast neutron image (b) of the Li-fueled reactor, post-operation. The flat and dark-field corrected radiographic image reveals a heat exchanger coil, the upper highly oxidized region (dark-gray), and the lower, primarily non-oxidized, elemental Li-rich portion (light gray). The image was taken over four minutes of exposure time. The red line represent roughly the beam size at the detector position.

The reactor vessel is a stainless-steel cylinder (OD $\sim 90 \mathrm{~mm}$, ID $\sim 75 \mathrm{~mm}$ ) with a large flange on top. The cylindrical reactor vessel contains a stainless steel heat exchanger coil near its base for reaction heat delivery to a compressed air stream. The reactor was filled $\sim 75 \%$ with $\mathrm{Li}$, evacuated to $\sim 200 \mathrm{~Pa}$, and externally heated to the auto-ignition temperature of $\mathrm{Li}$ and $\mathrm{CO}_{2}$ (approximately $410{ }^{\circ} \mathrm{C}$, above the melting temperature of $\mathrm{Li}$ at $180^{\circ} \mathrm{C}$ ). $\mathrm{CO}_{2}$ was then metered 
into the headspace above the molten $\mathrm{Li}$ pool, initiating the oxidation reaction. The reactor test was ended after the $\mathrm{CO}_{2}$ consumption rate dropped below the intake flow meter measurement range. After this test, the device was imaged by fast-neutrons.

Due to the very high Li content, the reactor would be fully opaque to thermal neutrons. X-ray images would be burdened by metal artifacts. Therefore, fast-neutron imaging offers the best approach to non-destructively imaging the reactor contents. A $3 \mathrm{~mm}$ thick ZnS/PP screen was used for the imaging. A fast neutron radiograph is shown in Fig. 1b. It reveals some internal metallic structures, including the heat exchanger coil and thermocouple wires. The reaction products (including $\mathrm{C}, \mathrm{Li}_{2} \mathrm{O}, \mathrm{Li}_{2} \mathrm{CO}_{3}, \mathrm{Li}_{2} \mathrm{C}_{2}$ [8]) all have larger attenuation coefficients than elemental $\mathrm{Li}$. As a result, the image is darker near the top, where the oxidation reaction reached high yield and more attenuating products are concentrated. This "crust" of products seems to have prevented $\mathrm{CO}_{2}$ from reaching the lower portion of the reactor. The lower portion of the reactor is much more transparent, suggesting the presence of unreacted elemental Li.


Figure 2: Segmentation (a. - horizontal cut, b.- vertical cut) and volume rendering (c.) of the internal contents of the Li reactor based on the tomographic reconstruction. The segmentation is based on three gray-level intervals: blue represents mainly elemental $\mathrm{Li}$, and green and red represent the reaction products and metallic parts inside the reactor.

We performed tomographic imaging of the reactor, acquiring 376 equally spaced projections as the sample was rotated $360^{\circ}$ about its vertical axis, and $3 \times 3$ pixel binning applied, resulting in a $317 \mu \mathrm{m}$ effective voxel size. At each projection angle, five one-minute long exposures of the object were taken and a pixel-wide median of the five images was applied to improve image quality and to suppress noise. The images were filtered for ring artifacts using the combined wavelet and Fourier filter by Munch et al. [10]. After reconstruction by Filtered Back Projection (FBP) the images were treated by an anisotropic diffusion filter [11] to de-noise them with minimal blurring. Figure 2 shows a segmentation of the reactor contents based on the reconstructed images (performed in VG Studio [12]). The segmentation was performed with three grey value intervals corresponding to increasing attenuation of the material (from blue to red). Blue represents mainly elemental $\mathrm{Li}$; green and red mainly represent the reaction products. Note that the metallic parts (heat exchanger coil, thermocouples) have sufficient attenuation to fast neutrons such that they are also segmented into the green band. This could be improved by a more thorough analysis and more sophisticated data segmentation methods, which is planned for future work. 


\section{Summary}

We have reported our efforts to apply a thermal neutron imaging beam line with a significant fast neutron component for fast neutron imaging. Studies were performed using the RAD beam line at the research reactor of the Budapest Neutron Centre. We have tailored the in-beam filtering of the beam line to provide as high as possible fast neutron flux while suppressing the gamma background and the thermal contribution. We have made a significant effort to optimize the imaging detector to achieve maximal efficiency, and achieved an imaging resolution of approximately $1.6 \mathrm{~mm}$. We demonstrated the utility of fast neutron imaging in the nondestructive analysis of robust and dense objects containing significant portions of both light and heavy elements. Such objects would be impossible to non-destructively image with other approaches. Tomographic images of a $\mathrm{Li}-\mathrm{CO}_{2}$ reactor ( $\sim 90 \mathrm{~mm}$ in diameter) were generated and presented.

\section{References}

[1] Fujine S., Yoneda K., Yoshii K., Kamata, M., Tamaki, M., Ohkubo, K., Ikeda, Y., Kobayashi, H., Development of imaging techniques for fast neutron radiography in Japan. Nuclear Instruments and Methods in Physics Research A 424 (1999) 190-199. https://doi.org/10.1016/S0168-9002(98)01326-6

[2] T. Bucherl, Ch. Lierse von Gostomski, H. Breitkreutz, M. Jungwirth, F.M. Wagner, NECTAR-A fission neutron radiography and tomography facility, Nuclear Instruments and Methods in Physics Research A, 651, (2011)86-89. https://doi.org/10.1016/j.nima.2011.01.058 [3] Zboray, R., Adams, R., Kis, Z., 2017. Fast neutron radiography and tomography at a 10-MW research reactor beamline. Appl. Radiat. Isot. 119, 43-50.

https://doi.org/10.1016/j.apradiso.2016.10.012

[4] Zboray, R., Adams, R., Kis, Z., 2018. Scintillator screen development for fast neutron radiography and tomography and its application at the beamline of the $10 \mathrm{MW}$ BNC research reactor, Applied Radiation and Isotopes 140 (2018) 215-223.

https://doi.org/10.1016/j.apradiso.2018.07.016

[5] Kis, Z., Szentmiklósi, L., Belgya, T., Balaskó, M., Horváth, L., Maróti, B., 2015. Neutron based imaging and element-mapping at the Budapest Neutron Centre. Phys. Procedia, 69, 40-47. https://doi.org/10.1016/j.phpro.2015.07.005

[6] St.Gobain, 2011. Organic scintillation materials.

[7] RC Tritec AG, T., 2017. Scintillators. 〈http://www.rctritec.com/en/scintillators.html〉.

[8] Greer, C. J., Paul, M. V., \& Rattner, A. S. (2018). Analysis of lithium-combustion power systems for extreme environment spacecraft. Acta Astronautica, 151, 68-79.

https://doi.org/10.1016/j.actaastro.2018.05.039

[9] ASTM, 2002. Standard test method for measuring fast-neutron reaction rates by radioactivation of nickel. E 264-02, ASTM International, United States.

[10] Munch, B., Trtik, P., Marone, F., Stampanoni, M., 2009. Stripe and ring artifact removal with combined wavelet - fourier filtering. Opt. Express 17 (May (10)), 8567-8591.

https://doi.org/10.1364/OE.17.008567

[11] Perona, P., Malik, J., 1990. Scale-space and edge detection using anisotropic diffusion. IEEE Trans. Pattern Anal. Mach. Intell. 12, 629-639. https://doi.org/10.1109/34.56205

[12] VolumeGraphics, 2018. VGstudio. 〈https://www.volumegraphics.com/en/products/ vgstudio.html> 